\title{
PROPERTIES OF FREAK WAVES INDUCED BY TWO KINDS OF NONLINEAR MECHANISMS
}

\author{
Aifeng Tao ${ }^{1}$,Jinhai Zheng ${ }^{2}$, Botao Chen ${ }^{3}$, Hui Li ${ }^{3}$ and Ji Peng ${ }^{3}$
}

\begin{abstract}
We investigate the dynamic and kinematic characteristics of freak waves using a direct phase-resolved nonlinear numerical method. The focus is on the understanding of the effects of different nonlinear wave-wave interactions on freak waves development and characteristics in the evolution process of modulated Stokes wave trains. Long time simulations of modulated Stokes wave trains, with different parameters, are obtained. Based on these simulations, we find that there are different kinds of freak waves in different time scales due to two kinds of different nonlinear mechanisms. One is the modulation instability and another related to the wave group interaction. Both the dynamic and kinematic characteristics of the different kinds of freak waves are distinct. Occurrence of freak waves (especially of large height) is usually correlated with broadband wave spectra.
\end{abstract}

Keywords: Freak Waves, Nonlinear mechanism, wave-wave interaction, modulation instability, wave group interaction

\section{INTRODUCTION}

Freak waves also can be called Rogue waves, first introduced by Draper(1965), are surface gravity waves whose wave heights are much larger than expected for the sea state. There are many definitions and definition approaches for this kind of disastrous phenomena. One common operational definition requires the wave height $\mathrm{Hf}$ of Freak wave should be at least twice the significant wave height Hs. Abundant observations or evidence of Freak waves and some potential physical mechanisms leading to their formation can be found in two recent reviews by Kharif \& Pelinovsky(2003) and Dysthe etc(2008). With the increasing number of terrible damages due to Freak waves, understanding the generation mechanism and characteristics of Freak waves has induced a considerable interest for both scientists and engineers.

Usually, Freak wave events can be explained by presence of nonuniform currents or bottom topography that may induce wave energy to focus in a limit area for reflection, refraction, and wave trapping. These mechanisms are well understood and may be explained by linear wave theory(e.g., Peregrine 1976; Lavrenov 2006). However, these reasons are not suitable to explain freak waves which occur in the open ocean away from nonuniform currents or influence of bathymetry. The well-known New Year Wave is a typical example of them. It is natural to associate the appearance of this kind of freak waves with the Benjamin-Feir instability (Benjamin\&Feri 1967). This instability, also known as modulation or sideband instability will result in focusing of wave energy in space and/or time as is shown by the experiments of Lake et. Al.(1977). Based on this idea, since the first work of Simith(1976), many authors(e.g., Trulsen 1996; Onorato 2002 etc.) tried to explain Freak waves by using of nonlinear Schrödinger equation(NLSE) and its generalizations such as the Dysthe equation. NLSE and Dysthe equation are the first and second term, respectively, in the hierarchy of envelope equations describing packets of surface gravity waves.

One cannot deny the advantages achieved by the use of envelope equations, including NLSE and Dysthe equations, which are always be used to describe the packets of surface gravity waves. Many authors, from this way, get a similar conclusion: nonlinear development of modulation instability leads to concentration of wave energy in a small spatial region. And they provide much valuable information about Freak waves. However, the terms of envelope equations, such as NLSE or Dysthe equations, cannot free themselves from two restrictions: narrow bandwidth and weak nonlinearity. At the same time most researchers agree that the occurrence of Freak waves is closely related with high

\footnotetext{
${ }^{1}$ Key Laboratory of Coastal Disaster and Defence of Ministry of Education,Hohai University, Nanjing, 210098, China

${ }^{2}$ State Key Laboratory of Hydrology-Water Resources and Hydraulic Engineering, Hohai University, Nanjing, 210098, China,

${ }^{3}$ College of Harbor, Coastal and Offshore Engineering, Hohai University, Nanjing, 210098, China
} 
nonlinearity. And for the uncertain mechanism of Freak waves, it is necessary to investigate the effect induced by bandwidth.

So far, the most direct and applicable way to study on the generation mechanisms and characteristics of Freak waves is a direct numerical solution of Euler equation. This solution can be made by the methods published in several well-known articles, Dold, Clamond and Grue, Zakharov. Here, we use High Order Spectra Method(HOSM), which are developed by Dommermuth\&Yue(1987).

The main purpose of this paper is to study on the generation mechanisms of Freak waves by computing and analyzing the properties of water wave long time evolution process. Based on a direct simulation numerical method HOS, a series numerical runs are performed varying initial weakly modulated Stokes wave trains, which are constructed by a Stokes carrier wave and sidebands or perturbations. There are three realizations are performed with different wave steepness. The sidebands are all adopted according to the most unstable conditions, which have been particularly addressed by Tao(2012).The evolution time computed here is much longer than $\mathrm{O}\left(T_{0} / \varepsilon_{0}^{-3}\right)$. Here $T_{0}$ and $\varepsilon_{0}$ are the time period and wave steepness of carrier wave. The definition of Freak waves applied here are the the common criterias

$$
\eta_{c} / a_{0}>2.5
$$

where $\mathrm{a} 0$ is the wave amplitude of carrier wave. Based on the numerical results, two different nonlinear water wave mechanisms present and the corresponding Freak wave are also different.

\section{BRIEF DESCRIPTION OF THE NUMERICAL APPROACH}

In order to get the direct numerical solution of Euler equation, the validated High Order Spectra method(HOSM) is applied in this study. The HOSM is a pseudo-spectral and Zakharov-equation-based method that follows the evolution of $\mathrm{N}$ wave modes and accounts for their nonlinear interactions up to an arbitrary high order, $\mathrm{M}$, in wave steepness. The method obtains exponential convergence with respect to both $\mathrm{N}$ and $\mathrm{M}$. By the use of the fast transform techniques, the method obtains an operation count that is (near) linearly proportional to $\mathrm{N}$ and $\mathrm{M}$. Due to its high efficiency and accuracy, the HOSM is an effective approach for long-time and large-space simulation of nonlinear wave-field evolutions. The details of this method and it's valuable applications in this field can be found in many reference, such as [Dommermuth\&Yue, 1987; Wu, 2004; Tao, 2007, 2010,2011, 2012 ].

\section{MODULATED STOKES WAVE TRAIN LONG TIME EVOLUTION}

In this section, we will investigate the characteristics of long time evolution processes initialized modulated Stokes wave train. The evolution process of narrow band modulated wave train, constructed by a Stokes carrier wave and imposed sideband according to most unstable modulation instability condition, will be studied firstly. Then the characteristics of extreme waves will be discussed to introduce the concept of new nonlinear mechanisms.

Initial condition with weakly modulated wave train

For the narrow band modulated wave train, the initial conditions we used are as following.

$$
\left.\begin{array}{l}
\eta(x, 0)=\eta\left[\varepsilon_{0}, k_{0}\right]+r_{1} a_{0} \cos \left(k_{-} x-\theta_{-}\right)+r_{2} a_{0} \cos \left(k_{+} x-\theta_{+}\right) \\
\phi^{s}(x, 0)=-\phi^{s}\left[\varepsilon_{0}, k_{0}\right]+\frac{r_{1} a_{0}}{\sqrt{k_{-}}} e^{k_{-} \eta} \sin \left(k_{-} x-\theta_{-}\right)+\frac{r_{2} a_{0}}{\sqrt{k_{+}}} e^{k_{+} \eta} \sin \left(k_{+} x-\theta_{+}\right)
\end{array}\right\}
$$

where $\eta(x, 0)$ and $\phi^{s}(x, 0)$ are respectively the free-surface elevation and potential of a right-going Stokes wave of steepness $\varepsilon_{0}$ and wave number $k_{0}$. Here $\varepsilon_{0}=k_{0} a_{0}$ and $a_{0}$ is the surface elevation of carrier wave. $k_{ \pm}=k_{0} \pm \Delta k$ and $\theta_{ \pm}$are wave numbers and phases of sideband respectively. Those parameters are chosen as the most unstable conditions in the initial period. Three cases are performed here. The parameters are listed in Tab.1. As an example, the initial wave relative amplitude spectrum and wave surface for $\varepsilon_{0}=0.09$ are shown in Fig.1. For all the cases, the calculation domain is $100 l_{0}$. The parameters for HOS are $\mathrm{M}=6, \mathrm{~N}=4096, \mathrm{~T}_{0} / \mathrm{dt}=64$. Here $\mathrm{dt}$ is the time step for simulation. 


\begin{tabular}{|l|l|l|l|l|l|}
\hline \multicolumn{7}{|c|}{ Tab.1. Parameters for modulated wave train } \\
\hline Cases & $\varepsilon_{0}$ & $\Delta k / k_{0}$ & $\theta_{ \pm}$ & $r_{1,2}$ & Evolution time $\left(t / T_{0}\right)$ \\
\hline I & 0.05 & 0.1 & $\pi / 4$ & 0.1 & 20000 \\
\hline II & 0.07 & 0.12 & $\pi / 4$ & 0.1 & 7000 \\
\hline III & 0.09 & 0.15 & $\pi / 4$ & 0.1 & 4000 \\
\hline
\end{tabular}
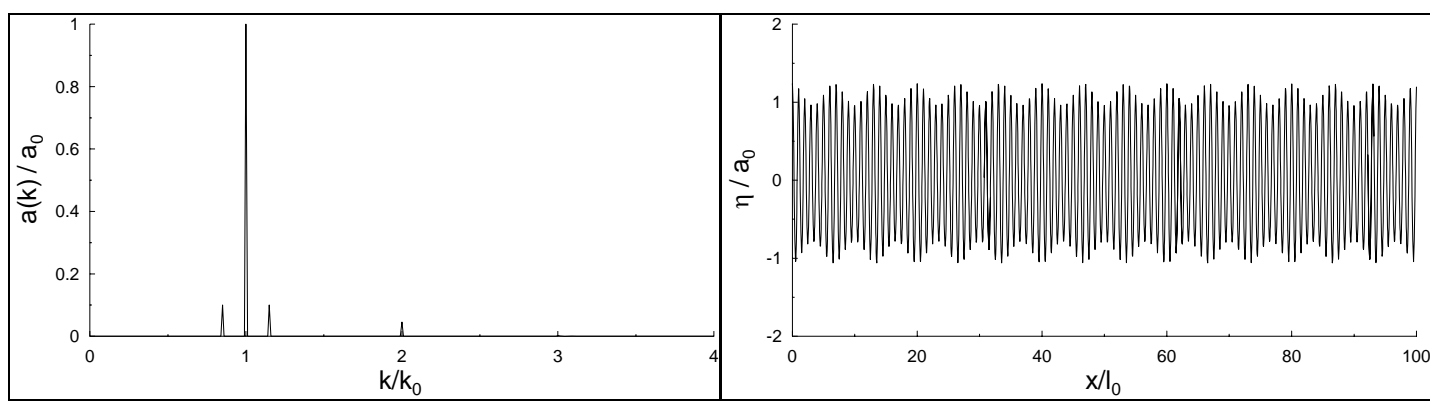

Fig.1. Initial wave relative amplitude spectrum (left) and wave surface (right) for $\varepsilon_{0}=0.09$

\section{Characteristics of Freak waves and the related mechanisms}

The variable $\eta_{\max }(t)$, shown in Fig.2, is the maximum of wave crests in the whole calculation domain at time $t$, i.e. $\eta_{\max }(t)=\max _{x}\left\{\eta_{c}(x, t)\right\}$. Then Fig. 2 shows the time evolution of the maximum amplification factor $A_{f} \equiv \eta_{\max } / a_{0}$ of the extreme waves. As a monitor index for the occurrence of Freak wave, $A_{f}$ will be used frequently in this paper. And the relationship between $A_{f}$ and some physical parameters, such as bandwidth, Kurtosis etc., will be discussed in the following. There are three pictures, which correspond to different initial carrier wave steepness, in Fig.2. From all this pictures, the modulation-demodulation recurrence induced by modulation instability can be seen clearly. However, after the time scale of $t / T_{0} \approx \mathrm{O}\left(\varepsilon_{0}^{-3}\right)$, the exact modulation-demodulation recurrence cannot happen again and the varying of Af presents chaotic or irregular characteristics. If we use $\eta_{c} / H_{s} \geq 1.25$ as Freak wave criteria and define $H_{s}=2 a_{0}$. It can be seen clearly that the maximum of Af will be enhanced with the increase of $\varepsilon_{0}$ and the occurrence probability of Freak waves will increase distinctly when $t / T_{0}>\mathrm{O}\left(\varepsilon_{0}^{-3}\right)$ for any $\varepsilon_{0}$.

There are two parameters always be used to describe the freak degree of the Freak waves. One is the local wave steepness $\varepsilon_{f}$ and the other is the ratio R between the extreme crest height and the nearest trough depth. Here, we adopt these two parameters in the following expressions:

$$
\begin{aligned}
\varepsilon_{f} & =k_{f} \cdot a_{f}=\frac{2 \pi}{L_{f}} \cdot \frac{H_{f}}{2}=\frac{2 \pi}{\left|x_{t r 1}-x_{t r 2}\right|}\left(\frac{\eta_{c}+\left|\eta_{t r 1}+\eta_{t r 2}\right| / 2}{2}\right), \\
R & =\frac{\eta_{c}}{\left|\eta_{t r 1}+\eta_{t r 2}\right| / 2}
\end{aligned}
$$

Here $\left(x_{t r 1}, \eta_{t r 1}\right),\left(x_{t r 2}, \eta_{t r 2}\right)$ are the coordinate of two troughs near the Freak wave crest. $L_{f}$ and $H_{f}$ are the local wave length and wave height of Freak wave.

As an example, two highest Freak waves correspond to two different time scales $\left(t / T_{0} \in[0,1000)\right.$ and $\left.t / T_{0} \in[1000,4000]\right)$ for $0=0.09$ are captured and those profiles are depicted in Fig.3. Some parameters for these two Freak waves are derived and listed in Tab.2. From Fig.3 and Tab.2, we know that the freak degree could be enhanced dramatically when the wave propagates for enough long time. It can be indicated from both the $\varepsilon_{f}$ and $R$. From Fig.3, it can be seen clearly that the Freak waves are different distinctly. The Freak wave present in the longer time 
duration is more like a water wall. Similar examples can be found easily in the other two cases ( $\varepsilon_{0}=0.05,0.07$ ), although the freak degree for them will be lower relatively.

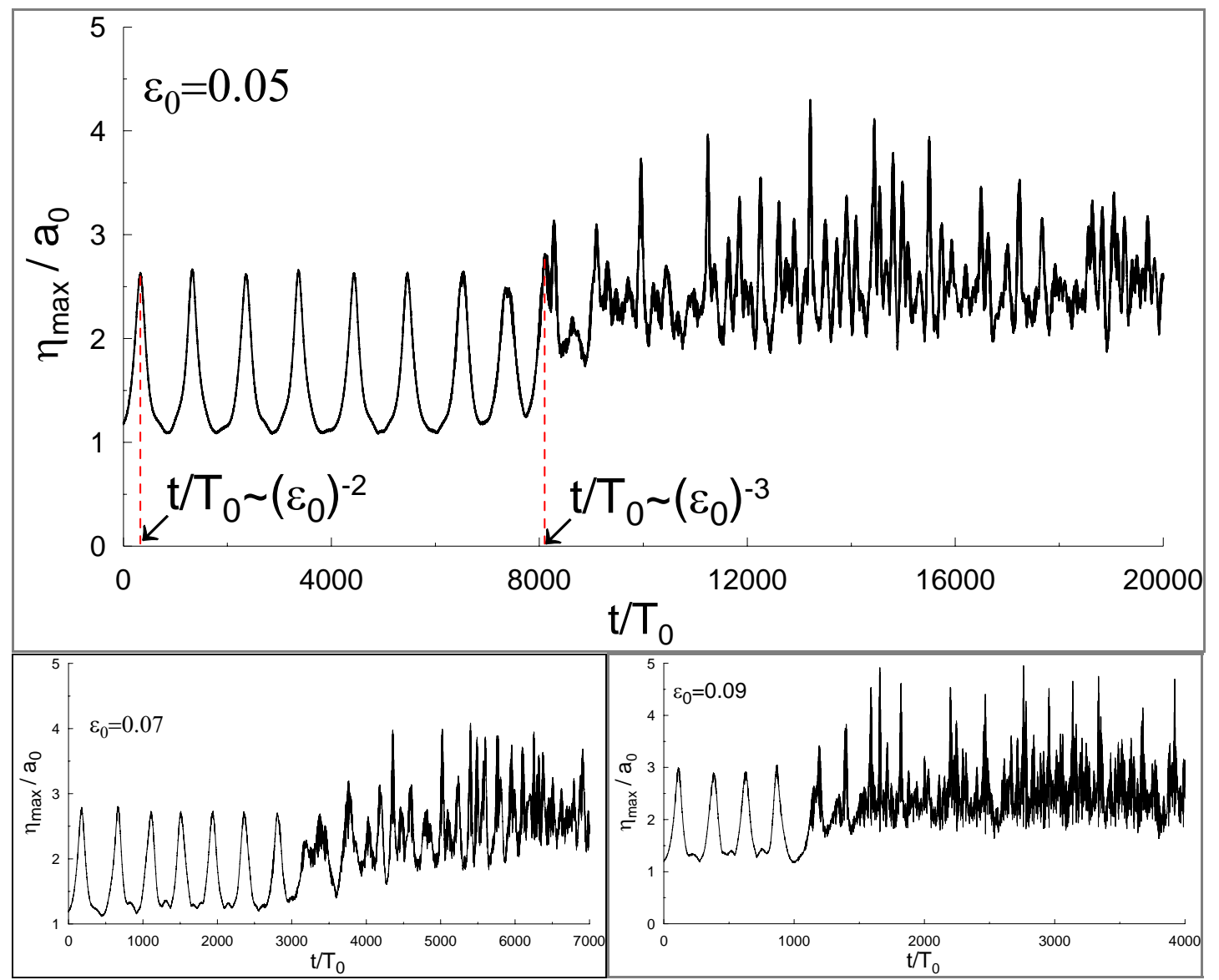

Fig.2. The evolution of wave surface maximum in whole computation domain with different initial carrier wave steepness.

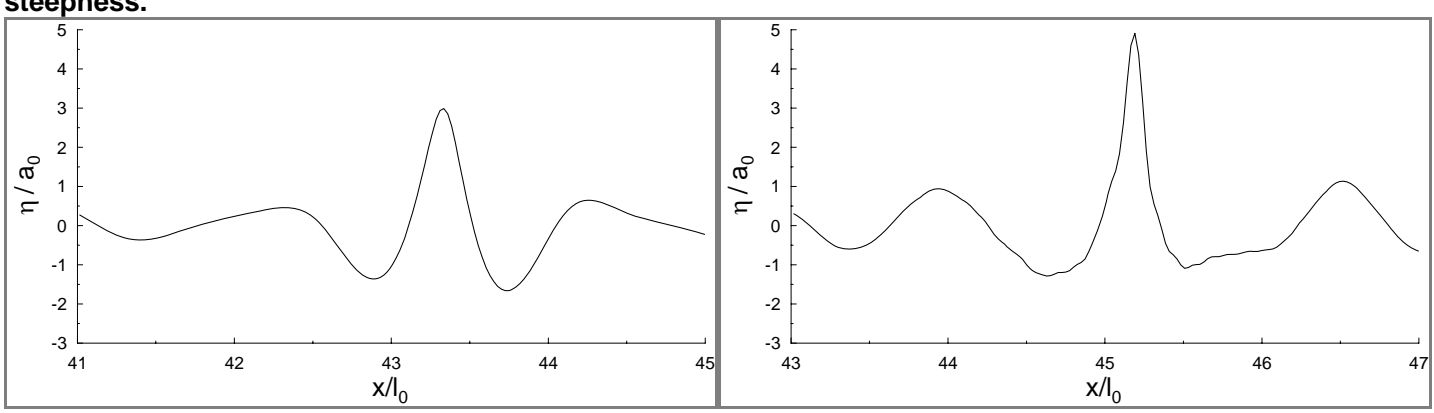

Fig. 3. Largest wave profile in different time. (The left one is captured at $t / T_{0}=110$ and the right one is captured at $\mathrm{t} / \mathrm{T}_{0}=1657$ for $\varepsilon_{0}=0.09$.)

\begin{tabular}{|l|l|l|l|l|l|}
\hline \multicolumn{7}{|c|}{ Tab. 2. Local information for Freak wave profiles in different time. } \\
\hline$t / T_{0}$ & $\left|\eta_{t r 1}+\eta_{t r 2}\right| / 2$ & $\eta_{c} / a_{0}$ & $H_{f} / a_{0}$ & $\varepsilon_{f}$ & $R$ \\
\hline 110 & 1.52 & 2.98 & 4.50 & 0.24 & 1.97 \\
\hline 1657 & 1.19 & 4.82 & 6.01 & 0.31 & 4.05 \\
\hline
\end{tabular}

It can be deduced that the modulation instability will take action rapidly along with the wave evolution process. It can reach its peak status at $t / T_{0} \sim \varepsilon_{0}^{-2}$ and then keep taking action as a dominated mechanism. However, when the modulated wave train evolutes to a further step $t / T_{0}>\varepsilon_{0}^{-3}$, more complex powerful nonlinear mechanisms will present rapidly. All the characteristics we addressed above are all in the physical domain. In order to make clear the new nonlinear mechanism, the wavelet transform is applied to two evolution processes, which are correspond to those two different Freak 
waves respectively. The results are shown in Fig.4. From this picture, the recurrence and nonrecurrence phenomena can be seen cleary. And the wave spectra is wider in the longer time scale $\left(t / T_{0}>\varepsilon_{0}^{-3}\right)$ compare to the time scale $t / T_{0} \leq \varepsilon_{0}^{-3}$. That means more new wave components get enery and join the evolution process. It need to be mentioned that the spectra is not continuous, although it looks like from the picture, since this is the numerical direcret results. It necessary to get the full pictures of the spectra, particularly at the time corresponds to those two different Freak waves, as shown in Fig.5.
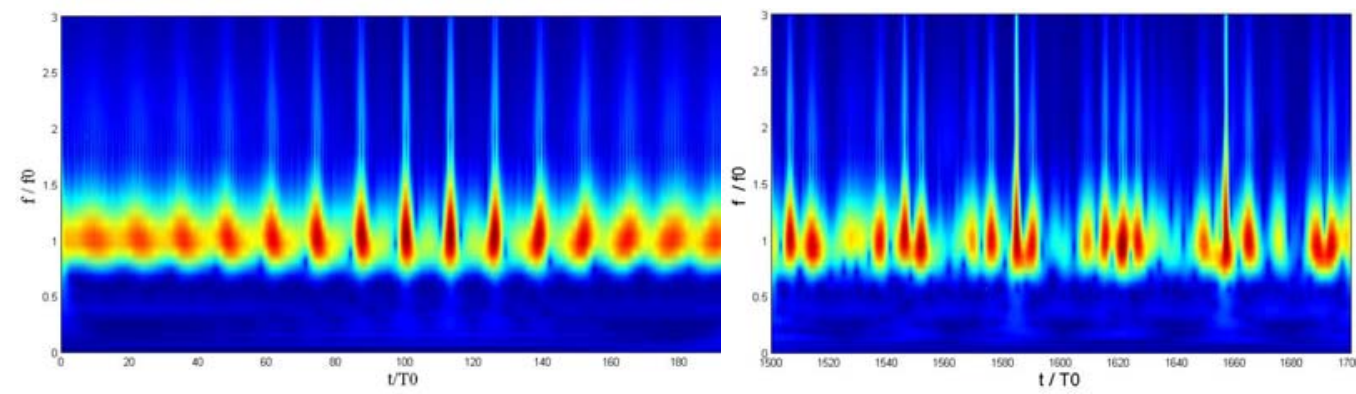

Fig. 4. Time series in frequcy domian are wavelet transformed. $\left(\varepsilon_{0}=0.09\right.$. $)$

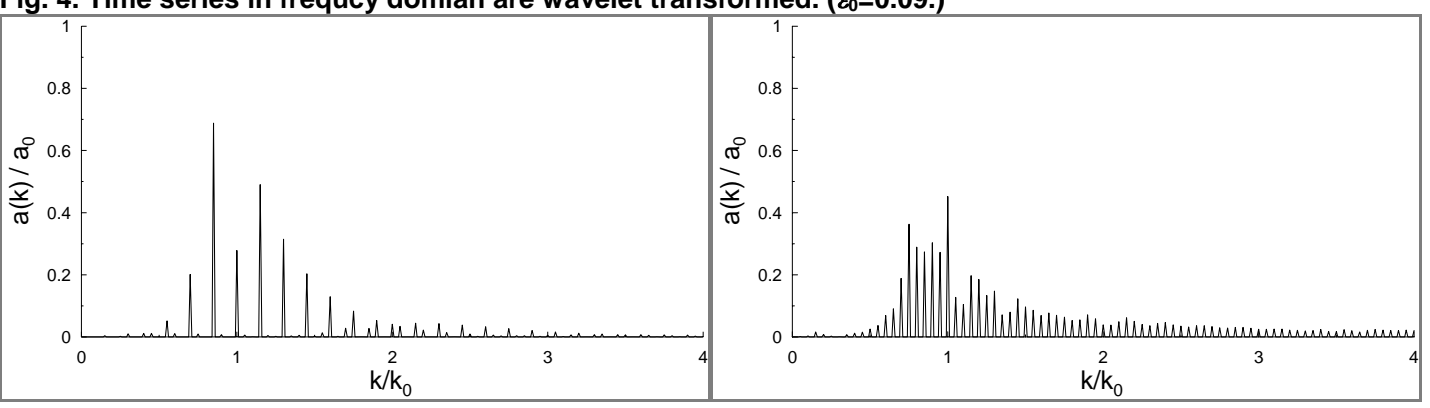

Fig. 5. Amplitude spectrum at different time for $\varepsilon_{0}=0.09$. (The time is $t / T 0=0,110$ and 1657 respectively, from left to right. )

Notably to see from Fig.5, comparing to the broaden of the spectra band in the longer time scale, more evident is the new wave components within the band. As desrcibed by Liu(2008), in the time scale $O\left(\varepsilon_{0}^{-3} T_{0}\right)$, the quintet resonance interaction presents and make appreciable change to the wavefield, but not the energy spectrum. This conclusion matchs our results very well. To a further step, more new wave componets mean more different wave group kinds can take action, as shown in Fig.6, wich are surface elevations at different time for $\varepsilon_{0}=0.09$. These pictures show that when the new wave modes are active, more and more different kinds of wave groups are formed. The interaction between the wave groups will be more intense and complex. And this kind of wave-groups-interaction might be the physical reason for the Freak waves, especially the extreme ones of Freak waves.

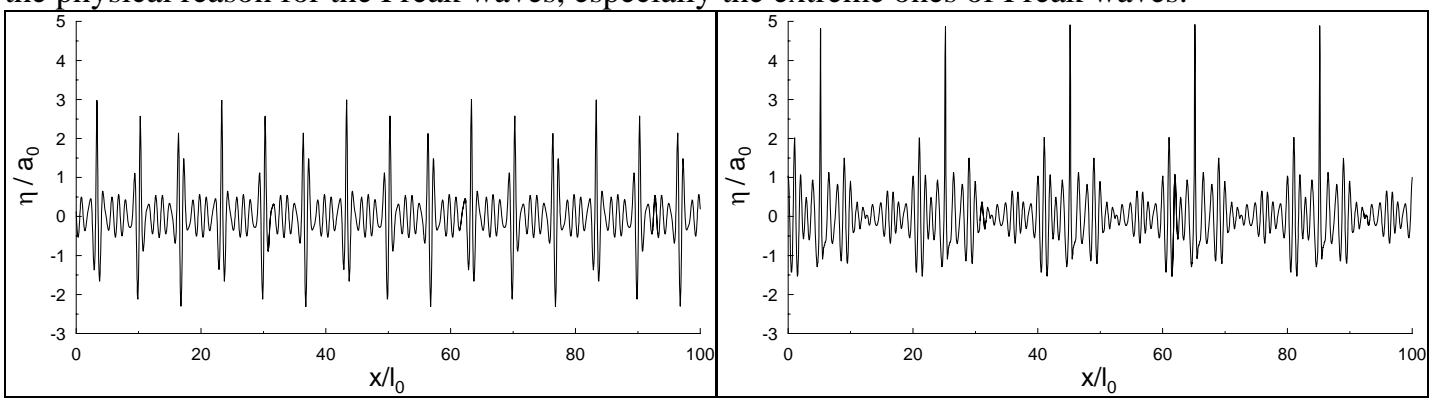

Fig. 6. Surface elevation at different time for $\varepsilon_{0}=0.09$. (The time is $t / T 0=0,110$ and 1657 respectively, from left to right. )

\section{CONCLUSIONS}

We consider a very long-time evolution of modulated Stokes wave trains, which is obtained using nonlinear HOS simulation (with order $M=6$ ). The steepness of the initial wave train is varying from $\varepsilon_{0}=0.05$ to 0.09 . Small disturbances at a broad band of wave numbers around the primary wave number are added at the initial time. In the initial period of evolution, modulation instability causes spreading of wave energy from the primary wave to its dominant sidebands and presents recurrence. 
As a result, large waves are developed in the modulated process and disappear in the demodulated process. After $t / T_{0} \sim \mathrm{O}\left(\varepsilon_{0}^{-3}\right)$, as broader and broader sidebands are developed, the recurrence is lost, and the wave-field becomes irregular. Importantly, the wave peak height of the developed extreme waves becomes much larger. There are typical two distinct mechanisms. One is the modulation instability, which will take effects as the dominate mechanism after $t / T_{0} \sim \mathrm{O}\left(\varepsilon_{0}^{-3}\right)$. The other can be interpreted as nonlinear wave group interactions heuristically, which might be the dominate mechanism in time scale $\mathrm{O}\left(\varepsilon_{0}^{-3} T_{0}\right)$.

\section{ACKNOWLEDGMENTS}

This work was supported by NSFC Grant no. 41106001, 509790335, 51137002, 51150110157 and JSNSF Grant no. BK2011026.

\section{REFERENCES}

Benjamin, T. B. 1967. Instability of periodic wavetrains in nonlinear dispersive systems. Proc. R. Soc, Lond, Ser. A, 299: 59-75.

Dommermuth, D. G., and Yue, D. K. P. 1987. A high-order spectral method for the study of nonlinear gravity waves. J. Fluid Mech., 184: 267-288.

Draper L. 1965. Freak’ocean waves. Marine Observer, 35:193-195.

Dysthe, K., Krogstad, H. E., and Muller, P. 2008. Oceanic rogue waves, Annu. Rev. Fluid Mech., 40: 287-310.

Kharif, C. and Pelinovsky, E. 2003. Physical mechanisms of the rogue wave phenomenon. European J. Mechanics/B - Fluid, 22(6):603-634.

Lake, B. M., Yuen H. C., Rungaldier, H., Ferguson, W. E. 1977. Nonlinear deep-water waves:Theory and experiment, Part 2, Evolution of a continuous wave train. J. Fluid Mech., 83: 49-74.

Lavrenov, I.V., Porubov, A.V. 2006. Three reasons for freak wave generation in the non-uniform current. European Journal of Mechanics B/Fluids, 25: 574-585.

Onorato, M., Osborne, A. R., Serio, M. 2002. Extreme wave events in directional random oceanic sea states. Phys. of Fluids, 14:L25-L28.

Peregrine, D. H. 1976 Interaction of water waves and currents. Ad. Appl. Mech., 16:9-117.

Tao, A. 2007. Nonlinear wave trains evolution and freak wave generation mechanisms in deep water. PhD Thesis, Hohai University, Nanjing, China (in Chinese).

Tao, A., Liu, Y. 2010. Rogue Waves Due To Nonlinear Broadband Wave Interactions, Proc. 25th International Workshop on Water Waves and Floating Bodies (IWWWFB25). Harbin, China, Paper No. iwwwfb25_41.

Tao, A., Zheng, J., Mee Mee, S., Chen, B. 2012. The Most Unstable Conditions of Modulation Instability. Journal of Applied Mathematics, 2012, Article ID:656873, 11 pages, doi:10.1155/ 2012/656873.

Tao, A., Zheng, J., Mee Mee, S., Chen, B. 2011. Re-study on recurrence period of Stokes wave train with High Order Spectral method. China Ocean Engineering, 25(4):679-686.

Trulsen, K., Dysthe, K. B. 1996. A modied nonlinear Schr odinger equation for broader bandwidth gravity waves on deep water. Wave Motion, 24:281-289.

Wu, G. 2004. Direct simulation and deterministic prediction of large scale Nonlinear ocean wave field. PhD Thesis, Massachusetts Institute of Technology, USA.

Liu, Y., Wu. G., Xiao, W., Yue, D.K.P. 2008. Nonlinear Wave Environments for Ship Motion Analysis. Proceeding of the 27th Symposium on Naval Hydrodynamics, Seoul, Korea 